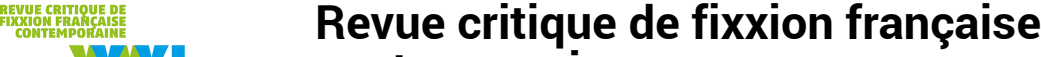 \\ WXI contemporaine
}

Radicalités

\section{Grouillements anarcho-poétiques}

Radicalité politique et expérimentations chez Antoine Boute

\section{Corentin Lahouste}

\section{(2) OpenEdition}

\section{Journals}

Édition électronique

URL : https://journals.openedition.org/fixxion/546

DOI : $10.4000 /$ fixxion.546

ISSN : 2295-9106

Éditeur

Ghent University

Référence électronique

Corentin Lahouste, "Grouillements anarcho-poétiques », Revue critique de fixxion française contemporaine [En ligne], 20 | 2020, mis en ligne le 15 juin 2020, consulté le 17 mai 2022. URL : http:// journals.openedition.org/fixxion/546; DOI : https://doi.org/10.4000/fixxion.546

Ce document a été généré automatiquement le 17 mai 2022

\section{cc) (†) $\odot$}

Les contenus de la Revue critique de fixxion française contemporaine sont mis à disposition selon les termes de la licence Creative Commons Attribution - Pas d'Utilisation Commerciale - Pas de Modification 4.0 International 


\title{
Grouillements anarcho-poétiques
}

\author{
Radicalité politique et expérimentations chez Antoine Boute
}

\section{Corentin Lahouste}

\author{
"On veut que les animaux réduits en émulsifs \\ pour les canons à eau de la police reprennent vie \\ et combattent auprès des manifestants sous \\ forme aquatique, polymorphe, immortelle." \\ (Fabien Clouette, Quentin Leclerc, Speedboat. \\ Manifeste pour une littérature révolutionnaire et \\ illimitée, Paris, Éditions de l'Ogre, 2019, p. 24.)
}

1 Dans un article consacré à la question de la perturbation et de la marginalité dans la littérature française contemporaine, Marie-Jeanne Zenetti note que

[d]ans le domaine de l'art contemporain, un important travail de construction de visibilité des productions perturbatrices a été mené [...]. Du côté de la littérature [...] ce travail n'a encore été amorcé que de façon relativement timide, et souvent en marge de l'institution. Les pratiques littéraires qui subvertissent les genres hérités de la modernité (performances, appropriations, etc.) font certes l'objet de questionnements, mais ces questionnements ont essentiellement lieu dans des sphères restreintes, qui ne bénéficient que rarement d'une importante légitimité institutionnelle, et au sein desquels les théoriciens sont souvent aussi producteurs. ${ }^{1}$

2 À l'aune de cette sagace allégation, et dans le cadre de cette intervention intégrée dans un dossier consacré aux radicalités littéraires, il convient effectivement de mettre en lumière et en valeur tout un pan de la littérature francophone contemporaine, la plupart du temps passé sous silence parce que, au-delà d'être minoritaire, recelant de textes excentriques, inclassables, déconcertants. Ces derniers viennent bouleverser tout de bon les pratiques scripturales et lecturales traditionnelles (c'est-à-dire liées au roman réaliste, qui constitue toujours et encore le modèle prototypique du roman contemporain), en déjouant notamment les principes de linéarité et d'unité à la fois narratives et discursives (unification, cohérence et univocité).

3 On s'appliquera ainsi, à l'occasion de cet article, à travailler et à analyser une œuvre particulière issue de ce déjà fort singulier corpus, qui, émanant de la littérature belge de langue française, y est elle-même excentrée, minoritaire - par rapport à “l'oligopole 
culturel parisien" ${ }^{2}$ : celle d'Antoine Boute (et de ses comparses ${ }^{3}$ en écriture diversifiée), qui se décrit comme un "poète expérimental travaillant à faire se chevaucher littérature, philosophie, performance et expériences sonores"4. Il s'agira de s'arrêter, plus spécifiquement, sur Opérations biohardcores ${ }^{5}$ et Apnée ${ }^{6}$, deux propositions poéticoromanesques récentes de l'auteur, résolument indéterminables, hétérogènes et éclatées, qui, dans leurs trames et en adoptant un ton fréquemment loufoque, invitent par ailleurs toutes deux à quitter le régime de la domestication (capitaliste), en "révolutionn[ant] le rapport à la vie" $(O B: 18)$ notamment "au niveau sensoriel intime" $(A: 132)$; qui sont, pour reprendre une formule d'un des deux livres, "politique[s] hors politique" $(O B: 61)$.

\section{Une radicalité politique affirmée}

4 Les œuvres d'Antoine Boute peuvent être très aisément appréhendées comme contestataires d'un certain ordre du monde: grosso modo, celui, actuellement prépondérant, régi par le capitalisme, le néolibéralisme et la bureaucratie : “ce qu'il nous faut c'est une révolution de la vie en direction de son noyau dur, débarrassée des addictions chiantes qui nous plombent l'existence" (OB: 49). Il y est clairement question de contester certaines structures et idéologies établies, et, spécifiquement, de se détourner de la "vieille vie néolibérale consumériste" (OB:54;A:57) et de son axiomatique utilitariste de l'intérêt, cette force présentée comme "vive et diaboliquement efficace mais [...] structurellement destructrice" (OB : 43), d'en finir, entre autres choses, avec "l'humanité rationnelle intelligente fière d'elle-même" ( $O B$ : 59) fonçant "droit dans un mur de catastrophes" (A:15). Dans un cadre idéologique en phase avec celui dressé par Anna Lowenhaupt Tsing dans son essai intitulé Le champignon de la fin du monde. Sur la possibilité de vivre dans les ruines du capitalisme (2017), ces textes, qui mettent fréquemment en scène des militants ou activistes en tous genres - notamment, une "militante écoféministe maraîchère anarcho-autonome" dans Opérations biohardcores ( $O B: 52)$ ou une bande "anarcho-déconstructiviste" dans Apnée $(A: 179)$-, se révèlent ainsi attachés à la valeur agonistique de la littérature ${ }^{7}$, comme en témoigne assez explicitement le titre d'un des deux romans où il est question d'opérations, terme qui renvoie à un imaginaire militaire, tactique. Dans leur visée de "révolution de la fiction hypnotique générale" $(O B: 70)$ et de récusation de tout régime de domination, ils s'inscrivent complètement dans la dynamique, qu'il est possible de qualifier de radicale ${ }^{8}$, également repérée et analysée par Marie-Jeanne Zenetti, de "revendication[s] d'une poésie de combat, pensée sur le modèle de 'l'action directe"'. Un de ses personnages, double de l'écrivain en tant que lui aussi "poète expérimental", affirme à l'avenant "réfléchi[r], en acte, aux impacts sur le réel du travail de la langue" (OB : 102, nous soulignons).

5 Une des singularités éthico-politique portées par les écrits du fantasque poète belge, dans lesquels il importe de "s'invente[r] une vie calquée sur la totalité cosmique" $(O B: 15)$, est qu'ils promeuvent une perspective contre-identitaire (de "défragmentation" ( $O B: 74)$ de l'identité) prônant un nomadisme identitaire, c'est-àdire une dissémination des êtres et une reconnaissance de leur côté pluridimensionnel. Il s'agit d'y mettre en avant le principe d'"identité-flux"10, suivant lequel cette dernière apparait comme toujours multiple et non lisse : "schizoïde"11, soit segmentée, éclatée, mue par de multiples turbulences, dessinant un domaine de l'inconstance, de la 
mobilité, de la précarité, et même de l'hétéroclite. Ces textes, qui viennent déjouer un rapport simpliste et grégaire à l'identité (très répandu dans une bonne partie des discours médiatico-politiques contemporains ${ }^{12}$, dans le "discours public structuré par les dirigeants politiques et [1]es propos et récits véhiculés par [de nombreuses] institutions étatiques"13), mettent de la sorte en lumière le fait que "nous vivons toujours au sein d'un pluralisme de mondes (opérationnels, mémoriels et fictionnels), à la fois superposés, décalés et hétérogènes entre eux" ${ }^{14}$, que tout être se trouve toujours "déchiré par diverses appartenances, divers intérêts, diverses idées de soi" ${ }^{15}$. Voilà comment un des personnages d'Opérations biohardcores assure vouloir "sortir la vie de ses gonds corporels trop précis" ( $O B: 32)$, tandis que la bande d'Apnée rencontre à un moment donné "l'inventeur d'une biologie parallèle casseuse du paradigme naturaliste séparant le corps de l'esprit" ( $A: 82)$. L'altération du soi et le brouillage des sens (voir $O B: 80)$ - "vivre autre, tout autre" $(O B: 84)-y$ apparaissent comme axiologie déterminante.

Dans la lignée de la dynamique désidentitaire, c'est également et plus encore une perspective antispéciste qui est déployée chez Boute. Y est posée une égalité radicale entre les différentes formes vivantes (humaines, animales, végétales) et même nonvivantes, minérales - comme un lac ( $A: 63-64)$ ou du cristal $(A: 141-147)$. On y repère un intérêt assez marqué pour les mondes et univers non-humains et les points de vue adoptés sont très ostensiblement non-anthropocentrés :

[J]e crois qu'il nous faut un peuple, mais pas qu'humain. Un peuple avec des animaux aussi, des chevaux des pigeons des corneilles plein d'insectes des loups des lynx des lézards tout ça, et des plantes, [...] un petit peuple expérimental discret des forêts et des zones sauvages. Un peuple invisible, qui non pas se fond mais nage dans le paysage. [...] Un peuple qui végète pas mal aussi, d'ailleurs, affalé, connecté à la façon des plantes, par des moyens un peu zarb, hormonaux, souterrains (OB : 49-50)

7 Ainsi, en ce qui concerne Opérations biohardcores, où l'on apprend dès la première page que les arbres sont considérés comme des "amis" ( $O B: 11)$, chaque chapitre consiste, à travers une narration à la deuxième personne (du singulier ou du pluriel), en une plongée dans une entité humaine, animale ou végétale, chacune s'agrégeant aux autres pour former petit à petit un "peuple le moins humain possible" (OB : 50). Le récit, formé de trois parties, est dès lors construit par interconnexions successives : chaque nouveau chapitre est établi à partir d'un élément secondaire - une des composantes du décor posé - du chapitre précédent.

Dans Apnée, il s'agit, de surcroit, pour le groupe d'individus se trouvant au cœur de l'intrigue, de disparaitre en tant qu'humains - c'est l'apnée recommandée par le titre du livre, "apnée de l'humanité urbaine trop humaine" $(A: 9)-$, pour laisser place à tout ce qui a été écrasé au cours de l'histoire et de l'évolution de l'humanité :

Allez hop il est temps de se rendre à l'évidence : la fête de l'humain moderne est finie, il est temps de discrètement disparaître. [La bande] postul[e] que soudain faire place nette, soudain laisser un max d'espace à la discrétion des autres formes de vie est la bonne et juste blague à faire au monde en ce moment. ( $A: 16$, nous soulignons) Soyons organiquement façonnables de manière à épouser les aspérités de ce qui constitue notre milieu. [...] Il nous faut sentir plus qu'agir afin d'envisager nos actions comme le simple prolongement du mouvement capté autour de nous. [...] Nos contours se diffusent et se diluent dans le vivant. On s'abandonne, on abandonne tout, on ne parle plus, on est absent, mais on existe. On a l'existence chaude, blotti au milieu de cette transparence. ( $A: 152$, nous soulignons) 
Cette perspective spécifique est déjà identifiable dans Opérations biohardcores, où il convient de "spéculer en transe en direction du végétal, de l'animal, de l'organique, même de l'infra-organique" ( $O B: 45)$, comme, par exemple, dans ce passage qui vient clore le sixième chapitre de la troisième partie :

Vous sortez tu te mets à courir, tu ne sais pas où tu vas mais tu ne veux plus voir d'humains de bagnoles de maisons, putain donnez-moi des paquets de feuilles en décomposition donnez-moi des flaques de la boue des étangs j'en peux plus je veux me laver là-dedans, tu cours tu bouffes le vent froid qui te brûle agréablement la gorge et ta copine te suit, elle gueule une sorte de cri proche du vomi, tu t'y mets aussi et tiens ça te fait un bien fou. (OB : 33)

10 Boute accrédite ainsi la dénomination du terrestre proposée par Bruno Latour ${ }^{16}$ pour parler des humains, non plus dès lors appréhendés comme supérieurs ou simplement séparés des autres êtres vivants partageant le même espace.

Ne pas dominer l'environnement dans lequel progressent les personnages des œuvres de Boute, mais s'y laisser couler, faire corps avec lui, être "vaporisable" ( $A: 153)$, "en précise connexion avec son milieu" ( $A: 67)$, tel est le programme politique que ceux-ci se donnent, ne voulant plus "prendre part à ce monde débile des humains contemporains" (OB:48). Une agentivité est donc fréquemment accordée à des animaux ou à des végétaux (par ailleurs absolument pas anthropomorphisés - à l'inverse d'une pratique fort répandue dans l'art et la littérature), mais l'enjeu sousjacent est plus ample que simplement représentatif: il est avant tout question d'engager à des transformations perceptuelles, de s'intéresser à l'infra-sensible, de chercher à (faire) vivre, pour reprendre les mots de Stéphane Vial ${ }^{17}$, d'autres ontophanies ouvrant à des qualia originales et singulières: "ce que l'on cherche c'est changer nos perceptions à vie!" $(A: 67)$. L'exemple le plus frappant est le passage d'Apnée consacré à un pigeon adopté par Freddo, double fictionnel du poète, qui est décrit comme un "chouette type, super intelligent" ( $A: 123$, nous soulignons) qui va dispenser tout un cours d'anthropologie à la petite bande insurrectionnelle, dans l'optique de leur transmettre "une nouvelle perspective à l'odorat, au toucher, à la vision" ( $A: 131)$.

Je vous propose que l'on laisse tous notre corps en monture à l'esprit prof-pigeon. Laissons-le habiter notre nerf optique, contaminer notre rétine, anamorphoser l'image formée, pour apprendre à travers eux ce qu'ils savent de la vie. Sortir le grand écran cérébral! Voir en panoramique! Enfiler des yeux de pigeon! Lâcher la stéréoscopie et le relief qui va avec! Grand vertige! Sentir la densité des cellules nerveuses s'intensifier, voir en quadrichromie! Décollage! Vision périphérique sur le monde humain!

Tout le monde part dans son trip, explore ce que nos parasites intimes ont à nous apprendre de nous-mêmes. Délire chamanisme pigeonesque. La plupart titubent bêtement en gloussant. Il y en a deux qui se filent des coups de tête pour avoir une fin de sandwich qui traîne là. Il y en a même un qui semble essayer de voler ou quelque chose du genre. La scène est bien ridicule, mais seulement en surface! La révolution se déroule silencieusement au niveau sensoriel intime! Ça parait stupide mais c'est très sérieux. (A:132, nous soulignons)

12 L'entreprise donnera lieu chez elles et eux à différents "acquis d'expérience" (A : 133-153) qui vont venir reconfigurer leur appréciation du réel - "modifier [leur] manière d'appréhender le monde" $(A: 149)$ - et induire des conjectures politiques originales, novatrices, liées, essentiellement, aux enjeux de la patience, de l'attention et de la passivité, d'une sensationnalité et sensualité puissamment aux aguets (que 
mettent en avant les illustrations accompagnant le texte), d'un érotisme et d'une corporéité pluralisés, flexibilisés.

13 L'on retrouve alors, au creux des textes de Boute, l'idée développée par Marielle Macé dans son essai Nos cabanes "d'honorer d'autres styles, d'autres pensées, d'autres comportements dans le sensible", de "suivre d'autres lignes qui constituent autant de propositions sur le monde et les façons de s'y tenir" ${ }^{18}$. Et l'on peut même dire, à l'avenant, que l'auteur-performeur belge encourage une perspective d'ensauvagement: le fait de passer, en prônant une polis élargie, d'une démocratie à une "biocratie" (OB : 90) radicale qui puisse faire entendre la diversité des clameurs du monde vivant. À la dévivification qu'entraine le capitalisme mondial "à la fois hyperfinanciarisé et violemment inégalitaire"19, il oppose la chaleur d'une sensibilité plurielle, joyeuse et palpitante, ouvrant des échappées et permettant ainsi de mieux respirer. L'horizon politique dressé et revendiqué est double: "au niveau macropolitique, sauver la planète, en transformant toutes ces forêts et ces terrains vagues en états indépendants non anthropocentrés, et, au niveau micropolitique, faire tendre la vie humaine vers son noyau hardcore dur, animal, végétal, matériel, nucléaire" (OB : 102). Et la conjecture nourrie par ces textes se révèle ultimement radicale dans le sillage d'un autre des sens de ce mot: en tant que relatif à la racine, à l'essence d'une chose. En effet, il s'agit de revenir à la fois à l'essence animale, naturelle, terrestre de l'être humain (vivant parmi les vivants), et à celle du politique/démocratique qui est, comme ont pu le souligner Jacques Rancière, Jean-Luc Nancy ou encore Daniel Bensaïd, fondamentalement anarchique $^{20}$ : "La politique n'a pas d'archè. Elle est, au sens strict, anarchique" ${ }^{21}$; "La démocratie implique par essence quelque chose d'une anarchie qu'on voudrait presque dire principielle"22.

\section{Une poétique anarchique}

14 Alors qu'il existe un paradigme anarchique dans la création littéraire contemporaine en langue française d'Europe occidentale (postérieure à Mai 68) ${ }^{23}$, venant engendrer une autre activation du terme "politique", voyons comment cette dynamique - qui valorise la mouvance contre le statisme, la multiplicité et l'hétérogénéité contre l'unitaire et l'uniforme, en générant un rapport au monde affranchi des modèles préétablis s'actualise dans l'œuvre d'Antoine Boute. Un de ses traits majeurs est la remise en cause du statut de l'auteur en tant que figure d'autorité souveraine, au profit, notamment, de la logique de collaboration artistico-poétique. Cette dernière est au cœur du travail poétique d'Antoine Boute qui a signé de nombreux ouvrages à plusieurs mains (notamment avec ses deux fils pour Les morts-rigolos (2014)) et qui ne cesse d'envisager la création comme un geste pluriel, un geste de rencontre(s), d'"interinteraction" $(A: 82)$. Par son travail et les publications qui en résultent - quasi toujours indéterminables, hétérogènes et éclatées -, il vient ainsi battre en brèche la vision romantique du créateur incréé, original, solitaire et singulier. La pratique poétique qu'il développe est d'emblée pensée comme ouverte, diffractée, à sans cesse remettre en jeu. Ses textes sont ainsi des lieux de voix multiples et croisées où est par conséquent récusée la perspective de propriété créative - un des éléments-phares de l'imaginaire (à tout le moins moderne) du livre traditionnel. En effet, s'y déploie une indécidabilité appuyée de l'instance auctoriale qui perd donc sa prééminence culturellement construite en Occident. Boute met ainsi en place un vrai processus 
d'incrustation, de perméabilité avec l'en-dehors, suivant lequel la notion d'auteur héritée du XVIII ${ }^{e}$ siècle, centrée sur l'individu, se voit déportée vers une appréhension ouverte au collectif, qui est assortie d'un enjeu politique appuyé, précédemment soulevé : une valorisation de la perspective désidentitaire :

Et donc amie lectrice, ami lecteur, ne vous étonnez pas si soudain dans ce livre boum on change de style, de rythme, d'énergie, de musique mentale et verbale... Haha! Voilà un des aspects fun de la blague[-livre]: elle s'écrit en temps réel par une tripotée de révolutionnaires de la disparition... Dans la bande ils écrivent le livre tous ensemble, la bande dans le livre dit je, je est la bande, c'est ça la blague, la blague politique de la bande, la bande est le dj d'une blague politique ! ( $: 22)$

On l'aura compris, les créations de Boute, qui sont marquées par une valorisation et l'investissement d'un régime dissensuel et déstabilisateur, battent en brèche la forme des récits classiques, tout autant qu'elles déclinent des formes textuelles génériquement indécises ${ }^{24}$, expérimentales. L'auteur belge et ses comparses revendiquent ainsi dans Apnée développer une "conception déviante [...] du concept d'écriture" $(A: 13)$ et présentent l'ouvrage comme "une blague-système chaotique de niveau 2" ( $A: 13)$, “UNE GROSSE BLAGUE FUMEUSE” ( $A: 14)$.

\section{CE LIVRE EST UN TROU ! ATTENTION PAS D'HISTOIRE! ATTENTION LA NARRATION \\ EST FOIREUSE! ATTENTION NOUS VOUS CONSEILLONS SI VOUS VOULEZ LIRE UN ROMAN TRANQUILLOU STRESSANT À SUSPENSE DE NE PAS LIRE CE LIVRE! CAR IL A ÉTÉ CONÇU SPÉCIFIQUEMENT POUR NE PAS CORRESPONDRE AUX CANONS DE LA VENTE ! NON ! CE LIVRE N'EST PAS CONFORME À VOS ATTENTES ! (A : 58-59)}

16 Avec Opérations biohardcores et Apnée, on se trouve ainsi du côté d'une pratique illittéraire, telle que théorisée par Bertrand Gervais ${ }^{25}$. Cet ancrage participe de la poétique anarchique propre aux textes de l'auteur qui font état d'une écriture sémiotiquement complexe, hybride. En effet, ses romans se voient amplifiés, pluralisés, par le recours à de nombreuses illustrations et dessins, de même que par des passages où s'étend une écriture manuscrite cahotante, tremblée (voir $A: 39,41,45,48-49$, 69-78), sans compter le travail typographique et de mise en page particulier qui y est maintes fois opéré. Dès lors, par moments, la dimension iconique y prévaut sur la dimension linguistique : s'y joue une "fragilisation de l'accès à la signification, du moins à cette partie de la signification du texte qui est associée à sa dimension langagière", pour reprendre les mots que Bertrand Gervais emploie afin de désigner une des modalités d'illisiblité propres aux productions illittéraires contemporaines et à leur "recherche tous azimuts des marges du texte et du sens" ${ }^{26}$. Chez Boute, la lecture est immanquablement bousculée ; les lecteurs et lectrices sont épisodiquement déportée-s aux limites de la textualité qui se caractérise par une visibilité exacerbée. À l'instar du roman de Reif Larsen, The Selected Works of T.S. Spivet, modèle du genre, "le texte devient une composante parmi d'autres d'une dynamique sémiotique plurielle, d'une part, et [...] d'autre part le livre, comme support de la textualité romanesque, devient visible et exploité dans sa dimension graphique" ${ }^{27}$.

17 Un autre élément de la poétique anarchique que l'on peut rattacher à l'œuvre de Boute se rapporte à un des quatre ${ }^{28}$ grands paradigmes narratifs de tout texte littéraire: le système des personnages. En effet, y est repérable une mise à mal de la logique du héros, de l'héroïque, ne serait-ce que parce qu'il est question de s'engager "[e]n avant pour une radicale, ludique, sérieuse et pertinente expérimentation de la sortie de l'humain" ( $A: 61)$. Aucun véritable personnage principal ne peut y être discerné : on n'y entrevoit pas de protagoniste prédominant autour duquel l'ensemble ou une grande 
partie des scènes s'articuleraient. Les propositions livresques de Boute et de ses comparses apparaissent bien davantage comme des narrations chorales où les points de vue et narrateurs-trices sont multipliés. Dans cette lignée, il n'est pas anodin que l'on distingue également dans Apnée des contenus glanés sur le web, tel que des notices tirées de Wikipédia (voir A: 17, 52-53), type de discours lui aussi non attribué/ attribuable à une seule personne. En substance, les créations poétiques de Boute, marquées par une esthétique du bricolage, rappellent que "[l]a vie sensible, au plus intime, est effectivement faite de régimes : régimes d'objets, régimes de relations, régimes d'attention, manières impropres et pourtant fermement qualifiantes, formes collectives qui font la vie de chacun et passent de l'un à l'autre, investissant un corps, puis l'autre, cherchant des corps" 29 .

18 C'est également la langue qui se révèle atteinte par la dynamique anarchique étudiée. En effet, au-delà d'ébranler la forme du récit, Boute dépoussière (et dérègle en partie) tout autant la langue commune, viciée par ce que Roland Gori nomme la "rationalité pratico-formelle marchande" ${ }^{30}$. Autant Opérations biohardcores qu'Apnée font droit à une joyeuse désinvolture dans l'écriture, au développement d'un ton débonnaire, souvent inscrit dans un registre familier et fortement imprégné de néologismes. C'est à une langue française très "décontractée" et oralisée (par certaines structures syntaxiques et lexicales ${ }^{31}$ ), qui intègre aussi bien de l'argot que de nombreux anglicismes, que les lecteurs-trices sont confrontée's. Celle-ci, informelle, tranche avec un style dit "littéraire", ainsi que le manifeste, parmi bien d'autres, ce dialogue tiré d'Opérations biohardcores:

- Allez je vous offre un café et vous me racontez vos soucis OK?

- Ça roule les couilles comme disent mes enfants, my God comme ils sont bien mes

enfants, ils me manquent tiens, merde c'est pas vrai je vais encore me mettre à

chialer!

- Non non racontez-moi plutôt votre métier, ça m’a l'air intéressant ça:

« Nécessaire, révolutionnaire mais trop destroy trash!» $(O B: 18)$

Il s'agit en réalité d'une langue très "affectivisée", qui possède une capacité expressive forte, où l'humour (de différents types: parodie, satire, grotesque régénérateur, autodérision, etc.) occupe une place centrale ${ }^{32}$. Il est perpétuellement question de faire vivre et vibrer un "enjeu rigolo/percussif" (OB : 19), à travers l'emploi d'un vocabulaire étonnant, déroutant.

Y est ainsi activée une contre-conduite langagière qu'un passage d'Apnée s'étendant sur plus de huit pages (A:107-115), où apparaissent de nombreux mots et segments verbaux échappant à toute compréhension directe, met particulièrement en valeur, comme en témoigne cet extrait qui évoque par ailleurs l'enjeu primordial d'une libération de l'écriture de ses schèmes trop normés :

HR RV rB jK kRJg kG JkRJ Gfaire corps avec son texte, et comment le lecteur est-il corporellement façonné dans sa lecture du texte ? uER u é Er èRt èk $\mathrm{K} J$ èr krk «fictions de monde» le texte joue-t-il? RuO pRO krLRK f kkJ JRLuI upQ p K eagencements qui ne soient pas nécessairement de l'ordre de la représentation, de l'assujettissement du visible au lisible compréhensible et signifiant. F F jkf RJ Rkt jrt iIouU Oo opPt tdomestication excessive de l'écriture par l'école. (A :113)

Par la langue quasi idiolectale qui est employée et qui inquiète par moments la lisibilité, Boute et ses complices en écriture mettent en effet sur pied une stratégie d'analphabétisation, afin de "donner naissance à une réalité autre, différente et plus 'sensée' que la réalité ordinaire", ainsi que pour "annuler les coordonnées normales et 
normatives du rapport à la réalité" ${ }^{33}$, comme le propose Antonio Bertoli dans son essai poétique Thérapie d'analphabétisation. Ils et elles développent ce que Jean-Marie Gleize nomme une "mécriture", c'est-à-dire une "subversion par la langue, [une] subversion de la langue, [une] transgression des codes, du code, [une] dissidence par dissonance, discordance, néologisme, cacophonie, dérégulation morphologique et syntaxique, excentricité verbale, grandes irrégularités" ${ }^{34}$. Les compositions présentées par Antoine Boute (parfois co-crées avec Chloé Schuiten) dans le cadre de l'exposition Dépli, accueillie par la Bibliotheca Wittockiana (Bruxelles) du 29 septembre au 3 novembre 2019, mettent également en évidence ce trait particulier de son travail poétique.

Nous terminerons par un dernier trait, lié au dispositif métatextuel des textes de Boute dans lesquels l'accent est davantage porté sur la démarche créative que sur l'œuvre (achevée) en tant que telle. Apnée peut en effet être appréhendé comme le récit d'une création, le livre mettant en scène sa propre rédaction, son élaboration progressive.

En plus de trouver des recettes l'ordi sert par ailleurs aussi à ceux qui veulent écrire le livre de la blague de la discrète disparition progressive : malin n'est-ce pas ? [...] Voilà un des aspects fun de la blague : elle s'écrit en temps réel par une tripotée de révolutionnaires de la disparition... $(A: 22)$;

Dans la suite de la blague l'ordinateur passe encore de mains en mains, c'est bien, ça permet chaque fois une vigueur autre! ( $:$ :97);

- Eh si on appelait le bouquin-blague La disparition de Freddo?

- C'est pas un peu facile facile? [...]

- Ou alors Postulant, recruter? Pour faire une suite avec S'enfonçant, spéculer et Inspectant, reculer?

- Ouais pas mal mais ça commence à devenir un peu lourdingue non, ces «-ant, er » à la fin vous ne trouvez pas?

- Apnée?

- Apnée?

- Oui, Apnée, pour dire la disparition biohardcore, c'est impossible et excitant comme l'apnée tu vois?

- Allez...

- Ou Printemps féral?

- Super mais « féral » personne ne connaît ce mot.

- Et alors? On est là pour apprendre non?

- Oui mais l'éditeur va encore dire qu'on ferme la porte plutôt que l'ouvrir ! (A : 104-105)

Boute et ses partenaires de création, en donnant l'impression que le livre que le lecteur ou la lectrice tient entre ses mains est comme en train d'être composé, ont ainsi recours à ce que René Audet a dénommé une théâtralisation du texte/livre: y est thématisée et figurée une textualité complexe, "de sorte que l'image du texte sort de son invisibilité conventionnelle au profit de sa démonstration" ${ }^{35}$. Le roman apparait ainsi comme "l'interface d'un monde dont il témoigne tout en en étant partie prenante" ${ }^{36}$. Si l'écriture devient par moments image(s), comme nous l'avons évoqué, c'est aussi parce que cela permet de figurer concrètement le geste d'écriture dans la textualité du livre. Comme a pu le développer Audet au sujet d'autres œuvres poétiques aménageant un procédé similaire,

[1]'adjonction d'images, d'écritures marginales, de pièces annexes complexifie certes le déploiement du monde fictionnel, mais ces éléments, au final, se substituent pour une certaine part au texte - ils accomplissent ainsi une partie du travail de description et de narration. L'autorité sémiotique du texte romanesque, supposée une et complète dans un livre au sens courant, se trouve ici partagée. ${ }^{37}$ 

dans de tel livres-objets (papier ou numériques), même si elle disperse en quelque sorte leur autorité, conduit pourtant à un renforcement de l'atmosphère propre à chaque œuvre ou, pour emprunter au vocabulaire des œuvres de réalité virtuelle, on dira qu'elle renforce leur pouvoir d'immersion" ${ }^{38}$. C'est donc une logique de présence - présenciation qui y est fortement à l'œuvre qui, sans même compter toutes les adresses aux lecteurstrices, génère la sensation d'une parole en acte, en pleine performance ${ }^{39}$, et, plus encore, impliquante, comme, lorsque dans Opérations biohardcores, est utilisée, tout au long du récit, la deuxième personne du singulier ou du pluriel, faisant en sorte que les lecteurs-trices ont non seulement l'impression de prendre part aux aventures relatées, d'y être assimilée's, mais sont même invitée's à les poursuivre en fin d'ouvrage :

Votre âge votre genre votre sexe sont indéterminés, vous êtes les lecteurs de ce livre, merci de votre attention et surtout n'hésitez pas à aller vivre dans les forêts et terrains vagues du monde, n'hésitez surtout pas à adopter la dégaine des révolutionnaires biohardcores [...] Enfin bref, la saison 2 est à vous. (OB : 129)

\section{Défiger, diversifier, évaser}

Les écrits d'Antoine Boute et de ses acolytes - de l'“Amicale biohardcore" (A:105) "dangereusement politique[s]" ( $A$ : 23), qui font notamment valoir la dimension opératoire du littérairo-poétique et s'appliquent entre autres choses à "faire la révolution du mot révolution" ( $O B: 49)$, appartiennent par conséquent à une frange de la littérature contemporaine qui réengage le politique dans le foisonnement de la vie, de la vraie vie, située, selon Alain Badiou, "au-delà de la neutralité marchande, et audelà des vieilles lunes hiérarchiques" ${ }^{40}$, en s'opposant tout particulièrement au capitalisme comme forme dominante des existences. À travers l'approche dividualiste, diversificatrice et désuniformisante, tout particulièrement marquée par le principe d'expérimentation, qu'il(s) met(tent) sur pied, est déployée une littérature non pas posée en majesté, mais évasée, enjouée, inscrite au cœur d'interactions de différents ordres - entre autres graphiques et visuels - qui viennent ouvrir la forme-livre, en la considérant avant tout comme un espace de rencontres plurielles, à la suite notamment de l'inscription du travail poétique dans un circuit de diverses performances et résidences ${ }^{41}$. De même, déjouer le côté figé que peuvent prendre les langues et les langages, syntagme que l'on retrouve dans Apnée ( $A: 113)$, constitue bel et bien un des principes centraux des créations de Boute et de ses comparses, cocasses, déstabilisatrices et dissonantes à plus d'un titre, en tant que "polyptiques poétiques plurimédiatiques" ${ }^{42}$, mais surtout que "flux d'intensité radicale" (OB : 28).

Il s'agit somme toute pour Boute, suivant une logique du grouillement et en reprenant une formule d'Evelyne Grossman, de radicalement élargir "le champ de nos perceptions et affects", en ouvrant à un "autre corps de sensations [...] à éprouver, à vivre, à penser" ${ }^{43}$, de sorte qu'il soit possible de se reconnecter au noyau dur de l'existence afin de la rendre "étonnante, profonde et sensible à tout moment" ( $O B$ : 99), et alors, en dernier ressort, qu'il soit également possible de se dire, tout imprégnéee par le "mysticoméditatif" $(A: 33)$ propre à ces textes : "purée la vie est quand même grande, belle et intense" $(O B: 12)$. 


\section{NOTES DE FIN}

1. Marie-Jeanne Zenetti, "Un art imperturbable ? Perturbation et marginalité dans la littérature française contemporaine", Proteus, $n^{\circ} 7$, Arts de la perturbation, Cécile Mahiou, Benjamin Riado (dirs), juillet 2014, p. 38-39.

2. Voir Jean-Marie Klinkenberg, "La production littéraire en Belgique francophone : esquisse d'une sociologie historique", Littérature, n44, L'institution littéraire II, 1981, p. 33-50.

3. Il s'agit, pour les deux textes pris en compte dans cette étude, de Chloé Schuiten, Clément Thiry et Jeanne Pruvot Simonneaux.

4. Voir deuxième de couverture d'Apnée.

5. Antoine Boute et Chloé Schuiten, Opérations biohardcores, Paris, Les petits matins, 2017 ; dorénavant $O B$.

6. Antoine Boute, Chloé Schuiten, Clément Thiry, Jeanne Pruvot Simonneaux, Apnée, Bruxelles, ONLIT éditions, 2018 ; dorénavant $A$.

7. La diffusion de certains extraits de ses textes sur le site d'information et d'analyse politique, philosophique et sociologique Lundimatin (https//www.lundi.am), au même titre que des textes de Nathalie Quintane ou Alain Damasio, marque de manière assez évidente cet ancrage spécifique et le caractère foncièrement politique, si ce n'est anarchique, de ses œuvres.

8. Une des acceptions du mot radical étant "qui révolutionne".

9. Marie-Jeanne Zenetti, “'Ce discours s'autodétruira dans quelques secondes' - exposition, contre-narration et montage documentaire", Raison publique, juin 2018, url : <https://raisonpublique.fr/1762/>.

10. Voir Andrea Semprini, La société de flux. Formes du sens et identité dans les sociétés contemporaines, Paris, L'Harmattan, 2003, <Logiques sociales>.

11. John Holloway, Changer le monde sans prendre le pouvoir. Le sens de la révolution aujourd'hui, trad. de l'espagnol par Sylvie Bosserelle, Montréal/Paris, LUX Éditeur/Syllepse, 2007, <Instinct de liberté/Utopie critique>, p. 207. Le penseur américain signale en ce sens que : "La conclusion à laquelle nous parvenons [à la fois nous sommes et nous ne sommes pas quelque chose] n'est un non-sens que pour la pensée identitaire, pour ceux qui pensent qu'être et ne pas être s'excluent mutuellement. La contradiction entre être et ne pas être n'est pas une contradiction logique, mais une véritable contradiction. Elle souligne le fait que nous sommes et ne sommes pas réellement réifiés, que nous sommes et ne sommes pas réellement identifiés, que nous sommes et ne sommes pas réellement classifiés, que nous sommes et ne sommes pas réellement désubjectivisés ; pour résumer, que nous sommes et ne sommes pas. Ce n'est qu'à la condition de comprendre notre subjectivité comme une subjectivité divisée et notre moi comme un moi divisé que nous pouvons donner sens à notre cri, à notre critique" (p. 206).

12. Voir notamment Romain Bertrand, Sylvain Laurens (dirs), "Identité(s) nationale(s) : le retour des politiques de l'identité ?", Savoir/Agir, 2007, n², p. 9-94.

13. Norman Wayne, "4. De la construction nationale à l'ingénierie nationale: l'éthique du façonnement des identités", dans Alain Dieckhoff (dir.), La constellation des appartenances. Nationalisme, libéralisme et pluralisme, Paris, Presses de Sciences Po, 2004, $<$ Académique>, p. 125.

14. Yves Citton, Médiarchie, Paris, Le Seuil, 2017, <La couleur des idées>, p. 92.

15. Tristan Garcia, Nous, Paris, Le livre de poche, 2018 [2016], <biblio essais>, p. 253.

16. Bruno Latour, Où atterrir ? Comment s'orienter en politique, Paris, La découverte, 2017, <Cahiers libres>, p. 110-111. 
17. Stéphane Vial, "Voir et percevoir à l'ère numérique : théorie de l'ontophanie", dans Mauro Carbone, Jacopo Bodini, Anna Caterina Dalmasso (dirs), Vivre par(mi) les écrans, Dijon, Les Presses du Réel, 2016, <Perceptions>, p. 77.

18. Marielle Macé, Nos cabanes, Paris, Verdier, 2019, p. 98.

19. Olivier Quintyn, Implémentations/Implantations : pragmatisme et théorie critique, Paris, Questions théoriques, 2019, <Ruby Theory>, p. 8.

20. Arthur Guichoux, dans son article portant sur Nuit debout et les "mouvements des places", souligne également que l'expérience démocratique "revêt une dimension an-archique, où s'indique l'absence d'arkhè, ou de principe directeur incontestable, qui régit et commande l'action des hommes, leurs manières de penser, d'être et d'agir ensemble" ("Nuit Debout et les 'mouvements des places'. Désenchantement et ensauvagement de la démocratie", Les temps modernes, $n^{\circ} 691$, Nuit debout et notre monde, novembre-décembre 2016, p. 50).

21. Jacques Rancière, Aux bords du politique, Paris, Gallimard, 2003 [1990], <Folio. essais>, p. 113 ; 150.

22. Jean-Luc Nancy, "Démocratie finie et infinie", dans Démocratie, dans quel état ?, ouvrage collectif, Paris, La fabrique, 2009, p. 85.

23. Corentin Lahouste, Figures, formes et postures de l'anarchique dans la littérature contemporaine en langue française du livre et de l'écran (Marcel Moreau, Yannick Haenel, Philippe De Jonckheere), soutenue le 15 mars 2019 à l'UCLouvain (Belgique).

24. On retrouve ici la dynamique non-catégorique propre à l'anarchique.

25. Voir Bertrand Gervais, "Imaginaire de la fin du livre: figures du livre et pratiques illittéraires", Fabula-LhT, n¹6, Crises de lisibilité, janvier 2016, url : <http://www.fabula.org/lht/ 16/gervais.html>.

26. Ibid.

27. René Audet, "L'objectification (visuelle, numérique) des romans, ou la narrativité à l'épreuve de l'expérience matérielle des œuvres", Textimage, $n^{\circ} 11$, Blessures du livre, Sofiane Laghouati, Andrea Oberhuber (dirs), p. 2, url: <http://revue-textimage.com/17_blessures_du_livre/ audet4.html>.

28. Les trois autres étant le temps, l'espace et la narration.

29. Marielle Macé, Styles. Critique de nos formes de vie, Paris, Gallimard, 2016, <nrf essais>, p. 238-239.

30. Roland Gori, La dignité de penser, Arles, Actes Sud, 2013, <essai>, p. 47.

31. Telles que "Ils sont chauds effrénés d'apprendre la vie" ( $A: 43)$, "Eh les gars dis-tu à ta famille, eh on dormirait pas là-bas? Regardez c'est grand, il y a plein de place et même une plaine de jeux trop cool, allez please venez on y va on s'installe là, c'est grand !" (OB : 100), ou encore "Bingo les gars! Pour pieuter ici y a no souçaille, vous avez frappé à la bonne porte, cet endroit, c'est du refuge 3 étoiles" ( $A: 144)$.

32. Il suffit de penser, entre autres très nombreux éléments, à l'importance du motif de la blague dans Apnée.

33. Antonio Bertoli, Thérapie d'analphabétisation. Poésie et essai, trad. de l'italien par David Giannoni, Bruxelles, Maelström reEvolution, 2010, p. 19.

34. Jean-Marie Gleize, "Opacité critique”, dans 'Toi aussi, tu as des armes'. Poésie \& politique, Paris, La fabrique, 2011, p. 39.

35. René Audet, "Diffraction. Pour une poétique de la diffraction des textes narratifs", dans Emmanuel Bouju (dir.), Fragments d'un discours théorique. Nouveaux éléments de lexique littéraire, Nantes, Éditions Cécile Defaut, 2015, p. 34.

36. Ibid., p. 36.

37. René Audet, “L'objectification (visuelle, numérique) des romans, ou la narrativité à l'épreuve de l'expérience matérielle des œuvres", art. cit., p. 4, url: <http://revue-textimage.com/ 17_blessures_du_livre/audet4.html>. 
38. Ibid., nous soulignons.

39. Comme, par exemple, ce qui se joue dans Le bavard de Louis-René des Forêts; voir Magali Nachtergael, "Le sens du récit", Acta fabula, vol. 8, n², mars-avril 2007, url: <https:// www.fabula.org/revue/document2958.php>.

40. Alain Badiou, La vraie vie, Paris, Fayard, 2016, <couvertures>, p. 52.

41. Voir, notamment, http://www.labellerevue.org/fr/critiques-dexpositions/ 2017/ biohardcore.

42. Formulation, quelque peu modulée, empruntée à Estelle Mouton-Rovira qu'elle utilise pour qualifier les livres d'Emmanuelle Pireyre.

43. Evelyne Grossman, Éloge de l'hypersensible, Paris, Minuit, 2017, <Paradoxe>, p. 16 et 25.

\section{RÉSUMÉS}

La présente contribution vise à mettre en lumière l'œuvre et le travail d'Antoine Boute (et de ses comparses en écriture diversifiée). En s'arrêtant plus spécifiquement sur Opérations biohardcores (2017) et Apnée (2018), deux textes poétiques (romans) indéterminables, hétérogènes et éclatés, on montre comment les écrits de l'auteur belge invitent à quitter le régime de la domestication, en révolutionnant le rapport à la vie notamment "au niveau sensoriel intime", mais aussi en prônant une dynamique désidentitaire et antispéciste. Il y est clairement question de se détourner de la "vieille vie néolibérale consumériste" et d'en finir avec "l'humanité rationnelle intelligente fière d'elle-même". Au-delà de leur contenu, qui les inscrit d'emblée dans une certaine radicalité politique - revendiquée au demeurant à de multiples reprises -, il s'agit également de mettre en valeur la manière dont Boute, qui se décrit comme un "poète expérimental travaillant à faire se chevaucher littérature, philosophie, performance et expériences sonores", déploie une littérature non pas posée en majesté mais inscrite au cœur d'interactions de différents ordres, entre autres visuels. On souligne en outre, en passant en revue toute une série de traits spécifiques (mise à mal d'une logique de l'héroïque ; propension récurrente pour l'abondance, la prolifération ; appétence pour le recours à l'humour sous différentes formes ; etc.), comment une poétique anarchique peut y être distinguée, qui réengage le politique dans le foisonnement de la vie, en s'opposant tout particulièrement au capitalisme comme forme dominante des existences.

\section{INDEX}

Mots-clés : poétique anarchique, expérimentations littéraires, littérature et politique, littérature belge

\section{AUTEURS}

\section{CORENTIN LAHOUSTE}

FNRS - UCLouvain 